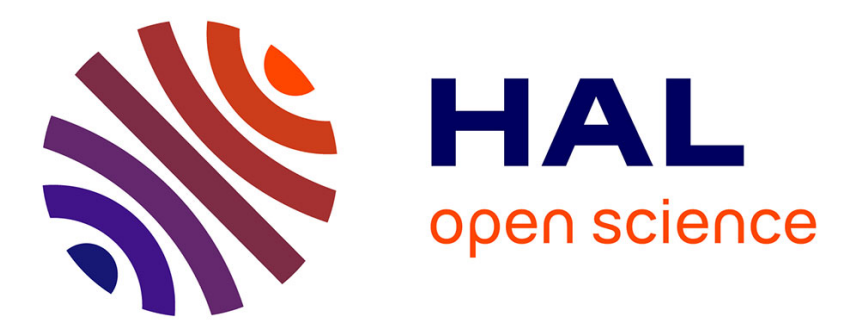

\title{
Head drop of a spatial turbopump inducer
}

Nellyana Gonzalo Flores, Eric Goncalvès da Silva, Regiane . Fortes Patella, Julien Rolland, Claude Rebattet

\section{To cite this version:}

Nellyana Gonzalo Flores, Eric Goncalvès da Silva, Regiane . Fortes Patella, Julien Rolland, Claude Rebattet. Head drop of a spatial turbopump inducer. Journal of Fluids Engineering, 2008, 131 (11), pp.111301. 10.1115/1.2969272 . hal-00266011

\section{HAL Id: hal-00266011 \\ https://hal.science/hal-00266011}

Submitted on 24 Feb 2020

HAL is a multi-disciplinary open access archive for the deposit and dissemination of scientific research documents, whether they are published or not. The documents may come from teaching and research institutions in France or abroad, or from public or private research centers.
L'archive ouverte pluridisciplinaire HAL, est destinée au dépôt et à la diffusion de documents scientifiques de niveau recherche, publiés ou non, émanant des établissements d'enseignement et de recherche français ou étrangers, des laboratoires publics ou privés.

\section{(c)(1)}

Distributed under a Creative Commons Attribution| 4.0 International License 


\section{Head Drop of a Spatial Turbopump Inducer}

A computational fluid dynamics model for cavitation simulation was investigated and compared with experimental results in the case of a three-blade industrial inducer. The model is based on a homogeneous approach of the multiphase flow coupled with a barotropic state law for the cool water vapor/liquid mixture. The numerical results showed a good prediction of the head drop for three flow rates. The hydrodynamic mechanism of the head drop was investigated through a global and local study of the flow fields. The evolution of power, efficiency, and the blade loading during the head drop were analyzed and correlated with the visualizations of the vapor/liquid structures. The local flow analysis was made mainly by studying the relative helicity and the axial velocity fields. A first analysis of numerical results showed the high influence of the cavitation on the backflow structure.
Nellyana Gonzalo Flores Eric Goncalvès Regiane Fortes Patella LEGI-INP,

Grenoble, BP53, 38041 Grenoble, France

\author{
Julien Rolland \\ CNES, Direction des Lanceurs, \\ Rond point de l'espace, \\ 91023 Evry, France \\ Claude Rebattet \\ CREMHyG-INP, \\ Grenoble, BP95, \\ 38402 Saint Martin d'Hères,
}

France

\section{Introduction}

Cavitation occurs frequently in the axial inducer stage of rocket engine turbopumps. It is initiated by a pressure decrease due to a high-speed local liquid velocity and can lead to a fatal failure in pump performance. In order to improve the design method and to evaluate the performance and application limits of inducers working under cavitating conditions, experimental and numerical works have been carried out by some research teams, as for example Refs. [1-8]. In complement to experimental observations, numerical approaches enable flow local analyses and the prediction of global performances.

Concerning three-dimensional (3D) numerical studies, cavitating flows in turbomachinery are generally modeled by a homogeneous fluid assumption through the one-fluid Reynolds-averaged Navier-Stokes equations (RANS). Different methods have been proposed to model the mixture and mass transfer between the liquid and vapor. We can cite the interface tracking method proposed by Hirschi et al. [9], the use of a state law to close the system $[3,4,10,11]$, the introduction of an additional equation including source terms for vaporization, and the condensation processes applied by Medvitz et al. [12], Ait Bouziad et al. [5,6], Mejri et al. [2,13,14], or Athavale et al. [15].

Although the numerical modeling of such a phenomenon has received a great deal of attention, it is still a very difficult and challenging task to predict 3D complex cavitating flows with an acceptable accuracy. Physical and numerical calculations should be improved and validated. Methodologies enabling 3D flow analyses and design criteria should be proposed.

In this context, the aim of the present study is to endeavor to improve previous works $[4,10,11,16]$ by

(a) carrying out complementary observations and obtaining new experimental data in the case of a three-blade inducer,

(b) applying and testing implemented physical and numerical models in the case of this inducer geometry running under different steady cavitating flow conditions, and (c) proposing an appropriate 3D analysis methodology.

The numerical simulation of cavitating flows in turbomachinery has been developed at the turbomachinery and cavitation team of Laboratoire des Ecoulements Géophysiques et Industriels (LEGI, Grenoble, France) in collaboration with the French space agency, Centre National d'Etudes Spatiales (CNES), and the rocket engine division of Snecma (Vernon, France), the civil and military aircraft manufacturer, a SAFRAN Group company. Studies are based on a homogeneous approach coupled with a barotropic state law to model the cavitation phenomenon [17]. The physical model has been previously integrated by the LEGI laboratory in the computational fluid dynamics (CFD) code FINETM/TURBO $[11,18,19]$, developed and commercialized by Numeca International (Bruxelles, Belgium). Experimental works have been conducted at the Centre d'Essais de Machines Hydrauliques (CREMHyG) laboratory (Grenoble, France). This paper presents obtained results concerning the head drop, blade load, power, and hydraulic efficiency evolution in the case of a three-blade inducer. Local analyses of the cavitation influence on secondary flows and backflow are also developed.

\section{Experimental Data}

The present study refers to a three-blade industrial inducer, CREM1, illustrated in Fig. 1. It was designed with the goal to suppress the rotating cavitation instabilities [16]. It is worth noting that, for this geometry, the nominal flow rate is much smaller than the zero-incidence angle flow rate.

Water tests were performed at CREMHyG. The TM2 bench is devoted to the characterization of high-speed inducers. Timeaveraged pressure measurements were performed at the shroud with two pressure taps, whose position is indicated in Fig. 2, connected to Druck 910-type pressure piezoresistive transducers with a relative accuracy of $0.2 \%$. The volumetric flow rate is measured by an automatic oil tool (AOT)-type turbine flowmeter, with a relative accuracy of $0.3 \%$. The rotational speed is measured with an HBM T32-type tachymeter with a relative accuracy of $0.1 \%$. From these measurements, the pressure coefficient $\psi$ and the cavitation parameter $\tau$ can be evaluated with an accuracy smaller than $0.4 \%$.

The inducer is placed in a transparent liner housing to enable 


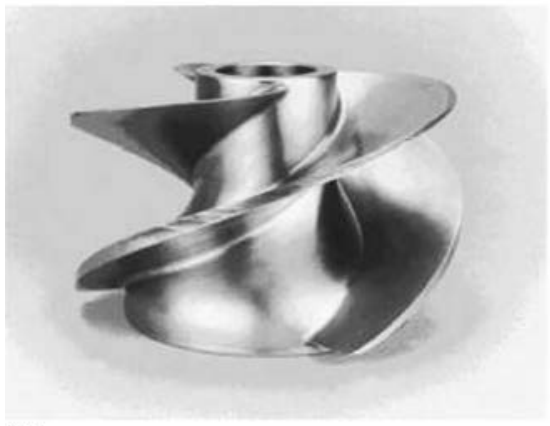

(a)

Fig. 1 Details of the inducer: (a) photograph of the inducer CREM1 and (b) a view of the inducer meshing

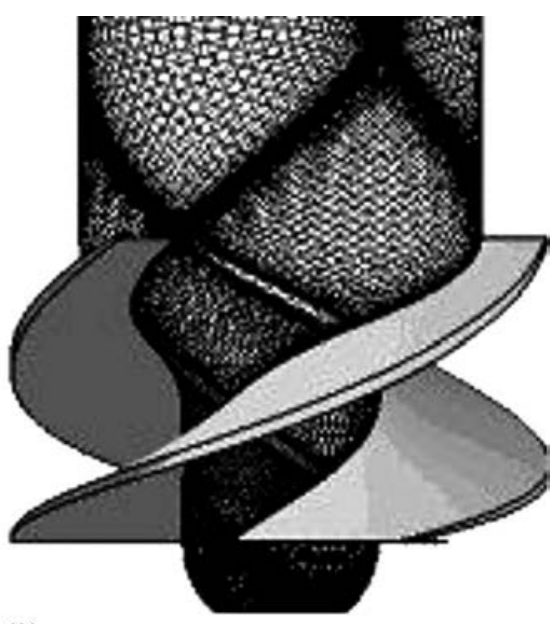

(b) the observations of the cavitating flow. Photographs and video movies are taken with stroboscopic light synchronized with the rotating shaft. These are used to observe the topology of vapor structures. Details of procedure and results are presented in Ref. [16].

A large range of flow rates was investigated around the nominal point of operation in noncavitating and cavitating conditions. Noncavitating experiments consist of setting a high static pressure level, to avoid any apparition of vapor and then to vary the flow rate. In cavitating conditions, the flow rate is kept constant, and the static pressure is decreased slowly to enhance vapor development in the inducer and to reach the performance breakdown.

In Fig. 3, breakdown curves are presented for three flow rates. Parameter $\Psi$ is evaluated from static pressures $P_{\text {inlet }}$ and $P_{\text {outlet }}$ measured at the shroud, as indicated in Fig. 2. $\tau^{*}$ is calculated as a function of the total pressure at the inlet level. The breakdown

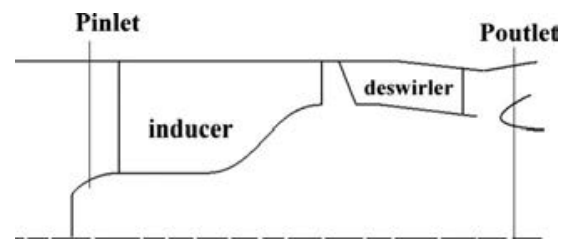

Fig. 2 Position of the pressure taps at the shroud used to measure pump performance (i.e., $\tau$ and $\Psi$ pressure coefficients)

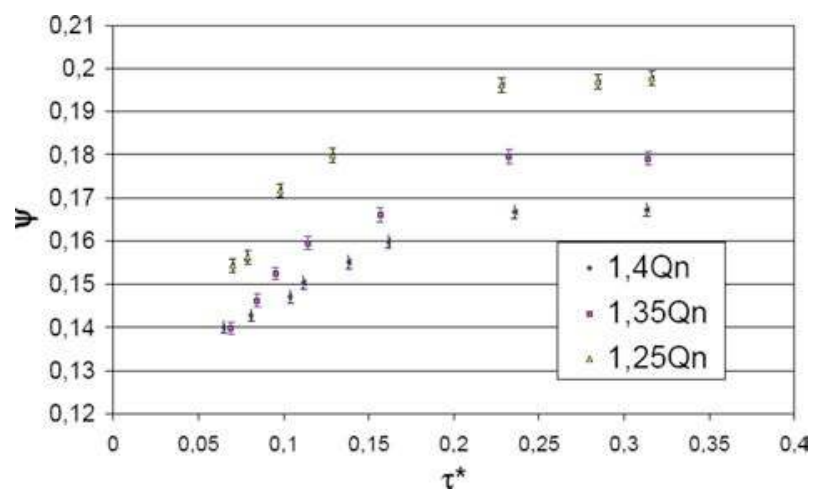

Fig. 3 Experimental head drop charts for the three different flow rates curves appear very gradual for this inducer geometry. Head drops equivalent to $\sim 10 \%$ of the noncavitating performance correspond to $\tau^{*} \sim 0.13$ (for 1.25Qn), and to $\tau^{*} \sim 0.11$ (for 1.35Qn and $1.4 Q n)$. In the final drop zone, corresponding to about $20 \%$ of the head drop and $\tau^{*} \sim 0.07$, each point is stabilized with $\pm 1 \%$ of the variation in the rate flow coefficient.

According to experimental observations [16], the cavitating zone is mainly located in a peripheral region and is fed by backflow circulation. From the spectral analysis of inlet pressures, and of axial and radial forces on the shaft, axial instability is observed during breakdown. No rotating cavitation signature appears on spectral cascades of the CREM1 inducer.

\section{Flow Model and Numerical Tool}

3.1 Governing Equations and the Barotropic Model. The applied two-phase flow model is based on a mixture assumption (one-fluid model). The compressible RANS system, written in a rotating frame, associated with the $k-\varepsilon$ turbulent model [20], has been solved. The barotropic model proposed by Delannoy and Kueny [17] is applied to model the cavitation phenomenon. In the model, $\rho_{1}$ and $\rho_{v}$ are, respectively, the density of the pure liquid (corresponding to $\alpha=0)$ and pure vapor $(\alpha=1)$. Both densities, corresponding to pure phases, are taken as constant. In the cavitation zone, the density of the vapor/liquid mixture is given as a function of the void ratio by the relation:

$$
\rho=\alpha \rho_{v}+(1-\alpha) \rho_{1}
$$

Incompressible liquid $\rho_{\mathrm{L}}$

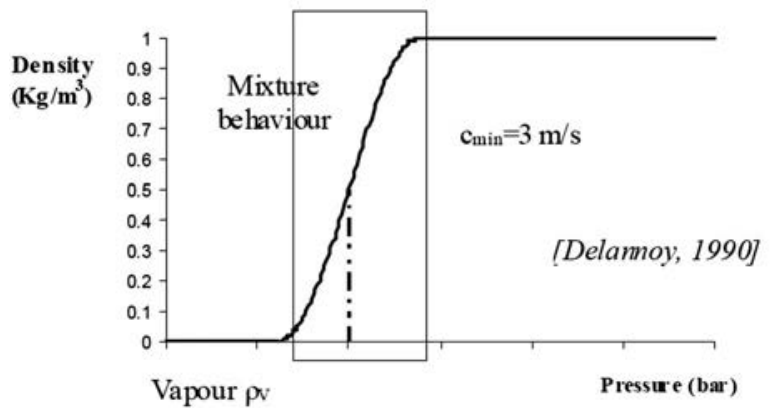

Fig. 4 The barotropic state law $\rho(p)$ for cold water 


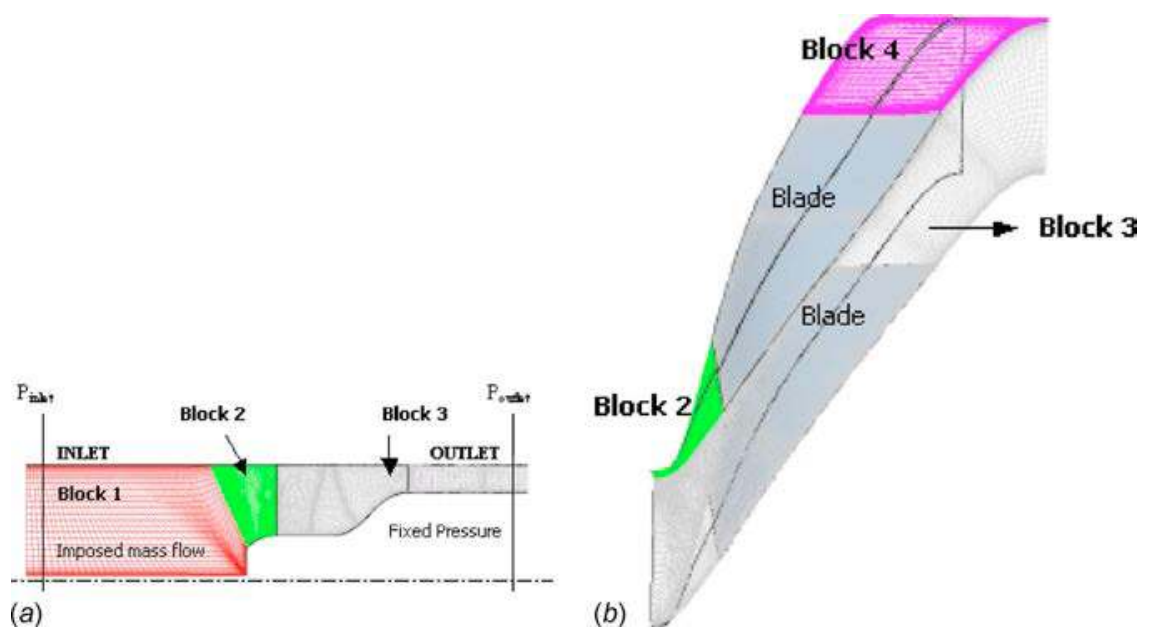

Fig. 5 Inducer views: (a) Inducer meridian view. Representation of mesh blocks and boundary conditions. (b) Representation of inducer blade-to-blade view mesh blocks.

This law is mainly controlled by its maximum slope, which is related to the minimum speed of sound $c_{\min }$ in the mixture. In all computations presented in this paper, $c_{\min } \approx 3 \mathrm{~m} / \mathrm{s}$ (Fig. 4). The influence of the law state parameter $c_{\min }$ on the behavior of the inducers has been evaluated in previous works $[11,20]$.

The liquid density is set to $1000 \mathrm{~kg} / \mathrm{m}^{3}$ and the vapor density is fixed to its physical value of $0.02 \mathrm{~kg} / \mathrm{m}^{3}$. Because of convergence difficulties in the case of the smallest cavitation numbers, some computations have been performed with a maximum value of $\rho_{v}=15 \mathrm{~kg} / \mathrm{m}^{3}$.

3.2 Numerical Methods. Steady numerical simulations were carried out using the 3D CFD code FINE ${ }^{\mathrm{TM}} /$ TURBO [19], developed by Numeca International in collaboration with the LEGI laboratory. The discretization is based on a cell-centered finite-volume approach for multidomain structured meshes. The convective numerical fluxes are computed with a second order central scheme stabilized by the artificial dissipation proposed by Jameson et al. [21]. An explicit four-stage Runge-Kutta time stepping procedure is used to advance the solution to the steady state. For incompressible areas, a low-speed preconditioner is introduced [18,23], enabling the reduction of the eigenvalue stiffness and the improvement of convergence.

The Yang-Shih $k-\varepsilon$ turbulent model [22] with extended wall functions is used. The turbulence transport equations are integrated with a first order upwind scheme. The physical model and the numerical code are detailed in Refs. [11,23].

In the proposed cavitation model, thermal effects are neglected in the vaporization and condensation phenomena [24]. Complementary works are in progress to improve the cavitation model in order to take into account thermodynamic effects $[25,26]$.

3.3 Grids and Boundary Conditions. Only one blade-toblade channel is considered and periodicity conditions are applied to its frontiers in azimuthal directions to simulate the presence of the contiguous blades. The mesh is composed of four 3D blocks (Fig. 5): the upstream part of the bulb, the blade-to-blade channel, and two blocks, respectively, upstream from the blade leading edge and downstream from its trailing edge. The whole mesh contains around 700,000 nodes for the first considered grid V1 (Fig. 1(b)). The tip leakage is not taken into account in the present study.

The $y+$ values from the center of the adjacent cells to walls vary from 0.8 to 100 , for all computations, and the largest $y+$ values are mainly placed in the leading edge near the shroud of the inducer.
The calculations are performed with water flow conditions. The mass flow is imposed at the inlet, and the static pressure is imposed at the outlet. The hub and the blades are rotating, the shroud is immobile. Wall functions are imposed along solid boundaries.

In order to develop the phenomenon of cavitation in the numerical simulations and to obtain different values of $\tau^{*}$ during head breakdown, the imposed vapor pressure is increased progressively. The calculated inlet pressure is used to evaluate the numerical cavitation parameter $\tau^{*}$. Parameter $\psi$ is calculated by the difference between the outlet and inlet static pressures, as indicated in Fig. 5.

In order to evaluate the grid influence, two additional grids (V2 and V3) have been tested. The grid specifications are shown in Table 1. Steady numerical simulations have been performed in cavitating conditions at $1.4 Q n$ flow rate with the same conditions applied in the reference grid (V1). Numerical head drop charts are

Table 1 Tested meshes

\begin{tabular}{lccc}
\hline \hline & Reference grid V1 & V2 & V3 \\
\hline Totals numbers of grid point & 697564 & 838672 & 962844 \\
$y^{+} \max$ & 97.6 & 87.2 & 78.3 \\
$y^{+} \min$ & 0.81 & 0.65 & 0.61 \\
\hline \hline
\end{tabular}

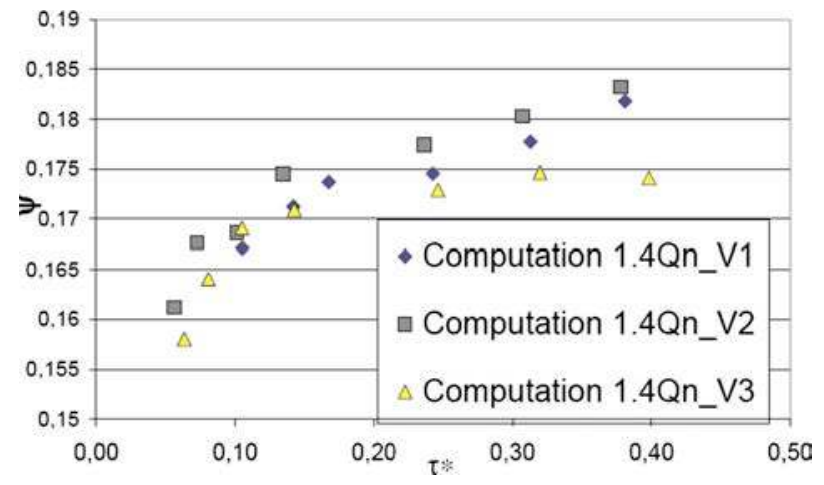

Fig. 6 Cavitation head drop curves obtained with three different grids at $1.4 Q n$ flow rate 

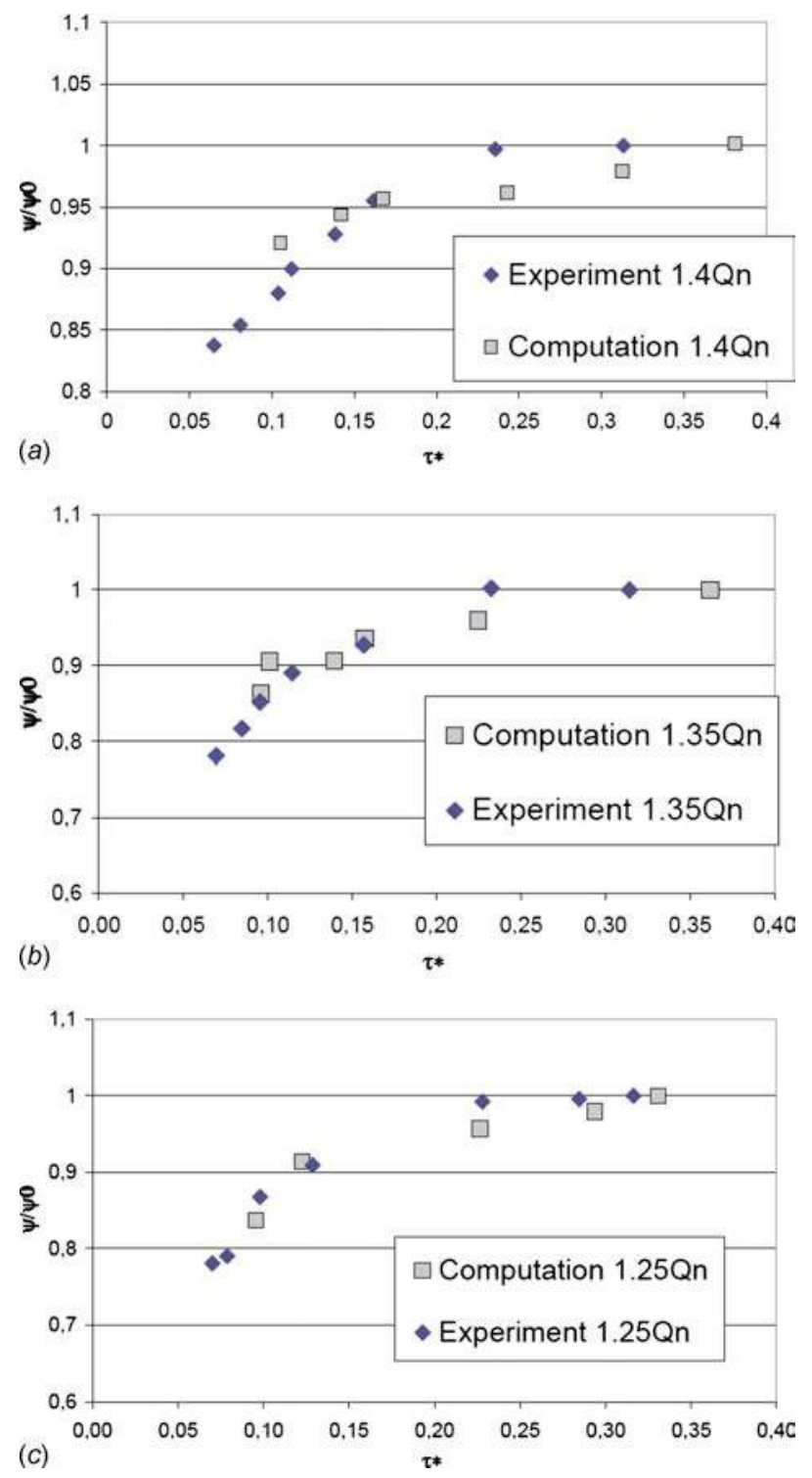

Fig. 7 Experimental and numerical head drop charts. (a) $1.4 Q n,(b) 1.35 Q n$, and (c) $1.25 Q n$.

presented in Fig. 6. It is worth noting that each calculated steady state solution was carefully converged.

The relative differences between noncavitating pump heads cal-

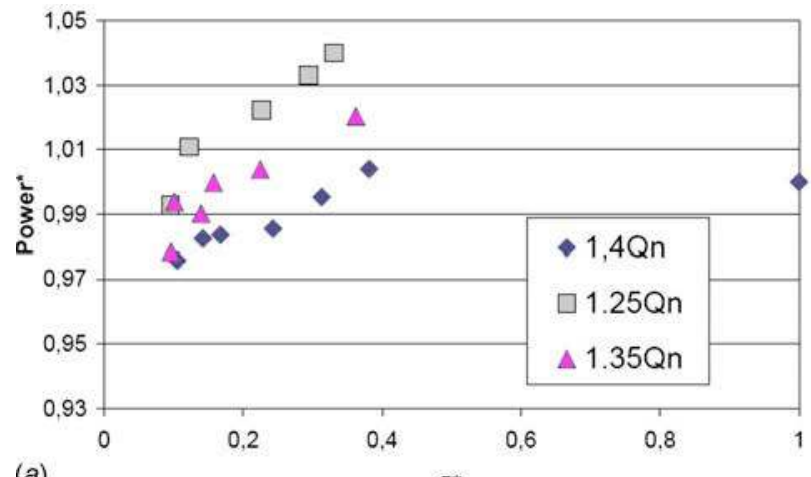

(a) culated with the three meshes are smaller than 5\%. Concerning $\tau^{*}$ equivalent to a $10 \%$ head drop, the maximum relative error between calculated values is about $30 \%$, which corresponds to a $\Delta t^{*} \sim 0.02$.

Numerical results have been analyzed in order to improve knowledge about head drop mechanisms, local flow characteristics, and secondary flows in the inducer. The development of cavitation structures (Sec. 4.4) calculated with these grids are quite similar, and the analyses of secondary flows, presented in Sec. 4.5, appear almost independent to the applied mesh.

The aim was also to propose a methodology of 3D calculations and analyses of complex cavitating flows. In relation to prior works presented in Ref. [11] concerning the inducer geometry, meaningful numerical work has been performed by Pouffary et al. [4], Numeca International, and Rolland [26] to improve the preconditioner and the stability of the numerical code for calculations of cavitating flows. It is worth noting that these numerical modifications led to major improvements in the accuracy and numerical stability in comparison to our previous computations [10].

\section{Numerical Results and Discussion}

Steady numerical simulations of the inducer have been performed with grid V1 in cavitating conditions at a given rotational speed and three flow rates: $1.4 Q n, 1.35 Q n$, and 1.25Qn. First, we present a quantitative analysis by comparing the experimental and numerical results of head drop charts.

The power and efficiency evolutions obtained by numerical simulations are illustrated for the three flow rates. Some qualitative results, concerning the blade load and visualizations of vapor/ liquid structures are also presented for several $\tau^{*}$ values. Finally, a study of secondary flows during the $\tau^{*}$ decrease is proposed.

4.1 Head Drop. Numerical head drop charts are compared with experimental ones for three flow rates in Fig. 7. The absolute comparisons between the experimental and numerical parameters $\psi$ are not possible because outlet pressure values are not taken at the same points (see Figs. 2 and 5). The head drop $\psi / \psi_{0}\left(\tau^{*}\right)$ is shown in relation to the maximal values $\left(\psi_{0}\right)$ obtained for each case (experimental and numerical) and for the different flow rates.

For $1.4 Q n$ and the flow rate, the numerical prediction at the $10 \%$ head drop were found for values of $\tau^{*}$ close to 0.1 . For $1.35 Q n$ and $1.25 Q n$ flow rates, it is close to $\sim 0.11$, these estimations correspond well to experimental observations. The most important differences between the numerical and experimental values of $\psi / \psi_{0}$ (around $\sim 4 \%$ ) are observed in the range of 0.2 $<\tau^{*}<0.4$.

We underline that for lower flow rates, the angle of attack of the flow at the blade leading edge is higher than for $1.4 Q n$; the cavity

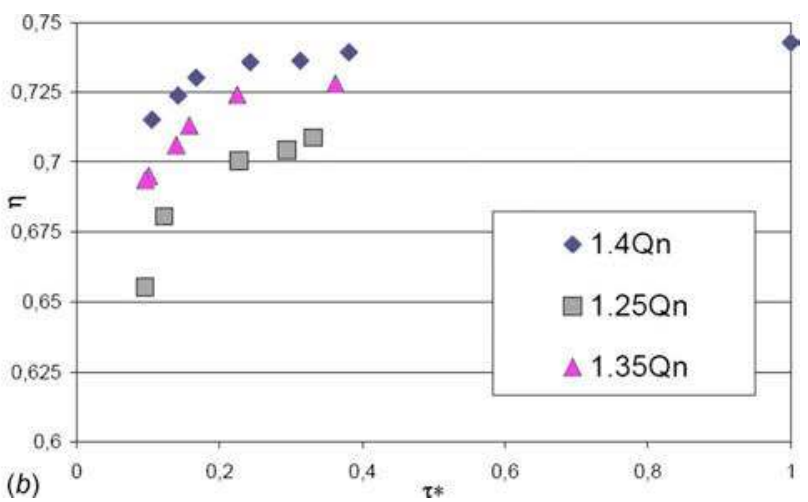

(b)

Fig. 8 Evolution of (a) the computational dimensionless available power as a function of the cavitation parameter $\tau^{*}$ and (b) the hydraulic efficiency as a function of the cavitation parameter $\tau^{*}$ 


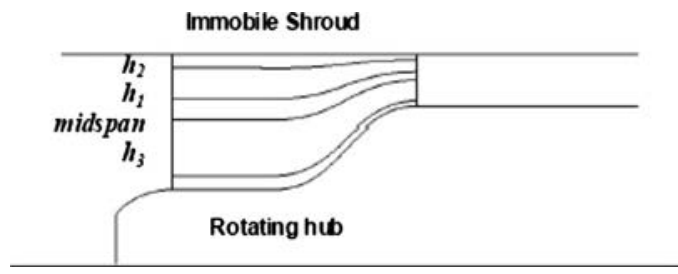

Fig. 9 Planes $h_{3}, h_{2}$, and $h_{1}$ and the midspan represented in the meridian view considered for the blade load analysis

thickness increases and sometimes it is difficult to obtain a good level of convergence.

Globally, the simulated head drops are gradual, and numerical results show a good correlation with the experimental ones for the three studied cases. The breakdown is clearly associated with a decrease in the torque and the hydraulic efficiency, as illustrated below.

4.2 Power and Efficiency Evolution. As illustrated in Fig. 8, the computed power and efficiency of the inducer are calculated at considered flow rates. The power is done from the calculated numerical torque and the fixed rotational speed. The torque is computed by the integration of the pressure and the stress tensor along the blade surface. The Power* represents the ratio of calculated power to reference power corresponding to the noncavitating condition for $1.4 Q n$. The efficiency is defined as the ratio of the provided power to the available power.

The maximal values of Power* are observed for $\tau^{*}$ around 0.38 , higher than the value in the noncavitating condition. This may be attributed to the change in the flow incidence angle due to the appearance of cavitation attached to the blade that induced a better blade load, mainly near the hub (see Sec. 4.3). For smaller $\tau^{*}$ values, the computed power decreases, this is attributed to the development of cavitation structures and a lower blade load.

The efficiency decreases with the cavitation parameter reduction. The efficiency variation is about $3 \%$ for $1.4 Q n, 4 \%$ for $1.35 Q n$, and more than $5 \%$ for $1.25 Q n$ in the range of analyzed $\tau^{*}$. We conclude that the head drop is associated with both the decrease in torque and the hydraulic efficiency.

4.3 Static Pressure Distributions Around a Blade Section. Figure 9 shows the different sections considered for the study of the static pressure distributions around the blade: midspan plane, two planes placed between the midspan and shroud, and one plane placed near the hub.

The blade load at midspan and three additional planes $\left(h_{1}, h_{2}\right.$, and $h_{3}$ ) at $1.4 Q n$ flow rate are drawn in Fig. 10 during the $\tau^{*}$ decrease. For these studied planes and analyzed $\tau^{*}$ values, it can be observed that the blades are loaded mainly near the leading edge. In the cavitating regime, the development of the sheet cavity from the leading edge of the suction side modifies progressively the blade load and, consequently, the inducer head decreases gradually, as illustrated in Fig. 7(a).

The blade load is slightly modified for $\tau^{*}=0.38$. For the smallest $\tau^{*}$ value, the figures show clearly a short sheet cavity attached from the leading edge of the suction side of the blades. For the considered conditions, the appearance of the cavitation does not influence the pressure side load. We do not observe the strong influence of the cavitation on the trailing edge pressure distribution because the cavity attached is not developed toward the interior of the blade-to-blade channels for the considered $\tau^{*}$ values.

The pressure values for plane $h_{2}$ (near the shroud) at the pressure side are higher than the ones evaluated for the other planes. Moreover, the blade load seems to be more important at the shroud.
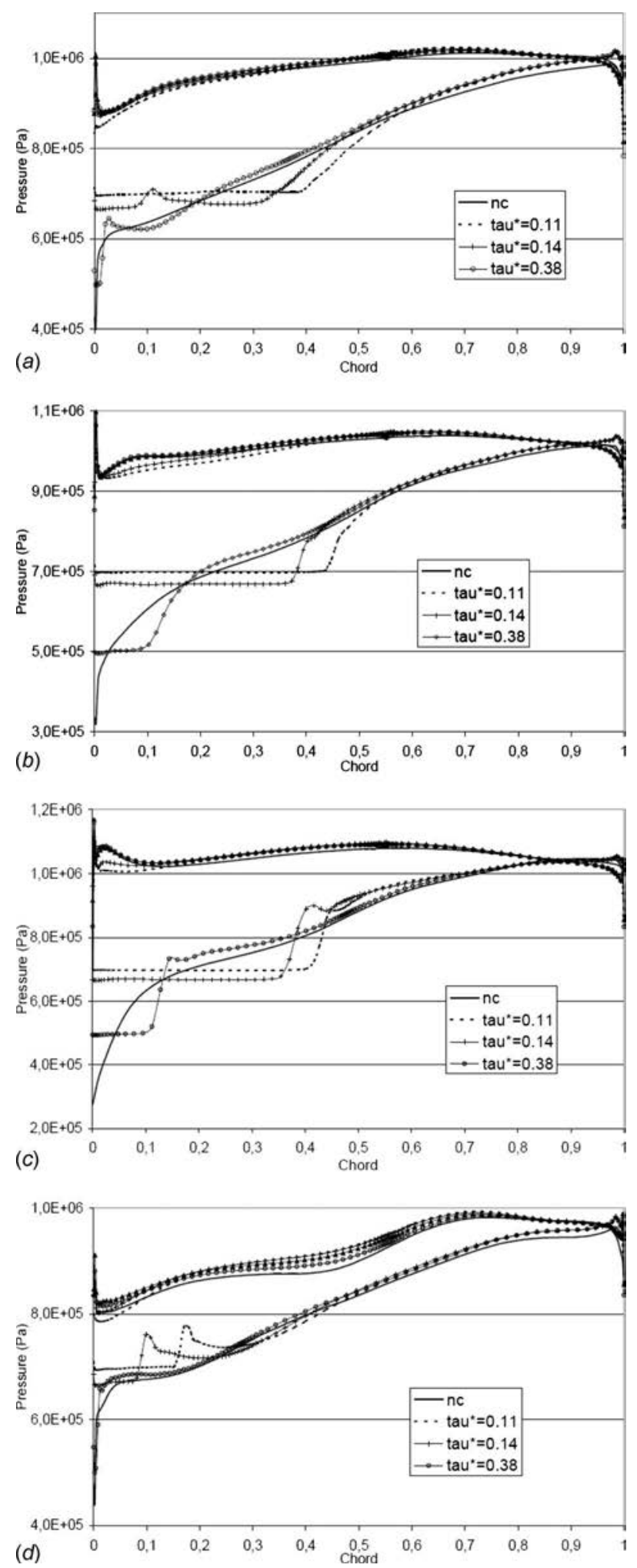

Fig. 10 Pressure distributions around the blade for decreasing $\tau^{*}$ at the $1.4 Q n$ flow rate in four locations: (a) midspan, $(b)$ $h_{1}$, (c) $h_{2}$, and (d) $h_{3}$

To improve the analyses during the $\tau^{*}$ decrease, the evolution of the blade load at additional plane $h_{3}$ near the hub (Fig. 10 $(d)$ ) has been studied. For this plane, a better blade load for $\tau^{*}=0.38$ was observed, if compared with the noncavitating condition; the appearance of the cavitation influences the pressure side load and 


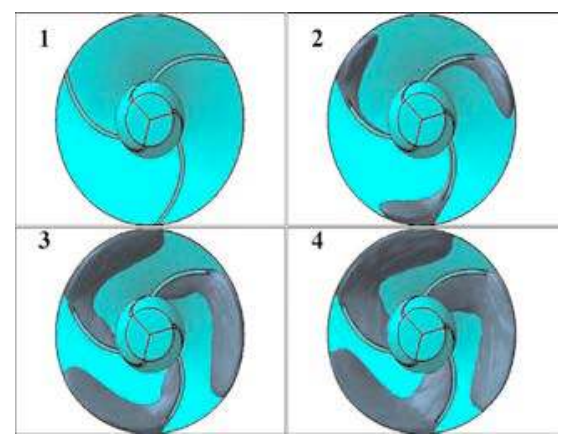

Fig. 11 Isoline of density $\left(\rho=950 \mathrm{~kg} / \mathrm{m}^{3}\right)$ during the $\tau^{*}$ decrease at $1.4 Q n ; 1$ is no cavitation, 2 is $\tau^{*}=0.31,3$ is $\tau^{*}=0.14$, and 4 is $\tau^{*}=0.11$
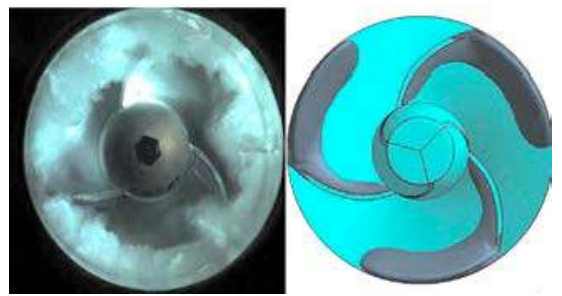

Fig. 12 Frontal view of the experimental and numerical results with an isoline of density $\rho=950 \mathrm{~kg} / \mathrm{m}^{3}$ for $\tau^{*}=0.17$ at $1.4 Q n$

modifies the trailing edge pressure distribution slightly. This behavior, observed near the hub, seems to explain the augmentation of the torque and the power (Fig. 8) for the $\tau^{*}=0.38$ value in comparison to the noncavitating case $(1.4 Q n)$.

4.4 Visualizations of the Vapor/Liquid Structures. The computed mixture vapor/liquid structures are presented in Fig. 11, during the $\tau^{*}$ decrease for the $1.4 Q n$ flow rate. The development of the cavitation structures are pronounced at the inducer periphery, and a large backflow structure in front of the blades is observed in Figs. 12 and 13.

The attached cavitation sheet slightly increases from the hub to the shroud and merges with the cavitating backflow structures. Head drop occurs progressively with the increase in vapor structures near the shroud. It is worth noting that at $\tau^{*} \sim 0.1$, the vapor structures do not reach the throat and do not enter into the bladeto-blade channel.

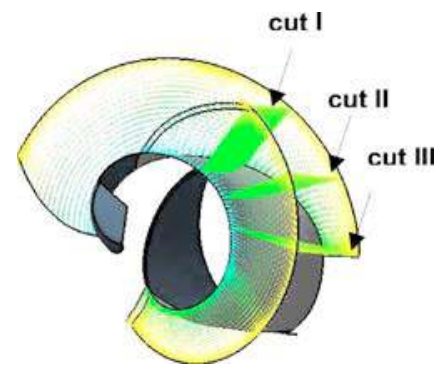

Fig. 14 Location of the analyzed flow sections in the inducer

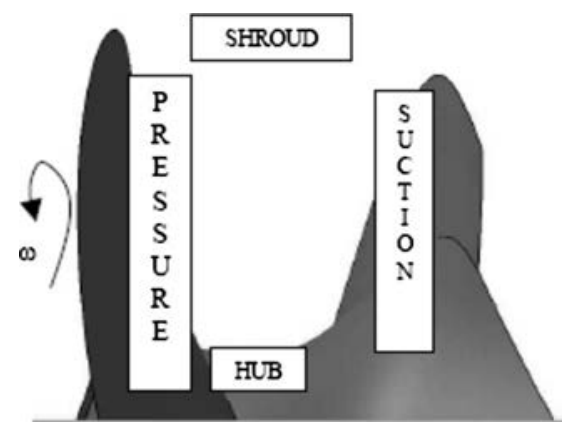

Fig. 15 Section representation for the local analysis of the secondary flow

The cavitation behavior obtained by numerical simulations presents a very good qualitative agreement with experimental visualizations (Figs. 12 and 13). We can observe that a consequence of the CREM1 design is the development of the ringed vapor structure at the shroud, which limits the development of the cavitation sheet on the blades.

Calculations performed with grids V2 and V3 lead to similar vapor/liquid structures.

4.5 Local Flow Analyses. An analysis of the flow within the inducer has been performed in three different sections in the blade-to-blade channel (Fig. 14). The local analysis of the influence of cavitation on the flow structure in the different sections has been made using the convention in Fig. 15.

4.5.1 Helicity. In the present paper, the analysis of the secondary flows is first based on the dimensionless relative helicity.

Secondary flows can be characterized by the dimensionless relative helicity, which is defined as

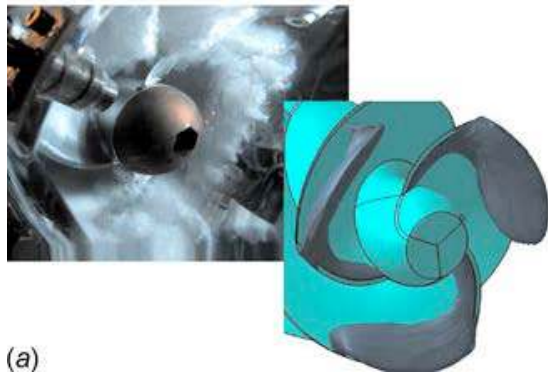

(b)

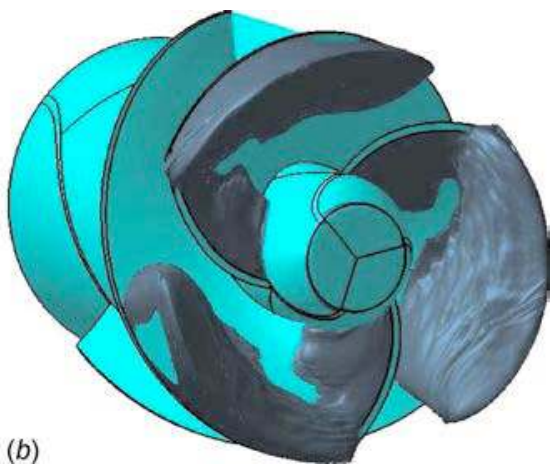

Fig. 13 Lateral view of (a) the experimental and numerical results with an isoline of density $\left(\rho=950 \mathrm{~kg} / \mathrm{m}^{3}\right)$ for $\tau^{*}=0.17$ at $1.4 Q n$ and $(b)$ the numerical result with an isoline of density $\left(r=950 \mathrm{~kg} / \mathrm{m}^{3}\right)$ for $\tau^{*}=0.08$ at $1.25 Q n$ 


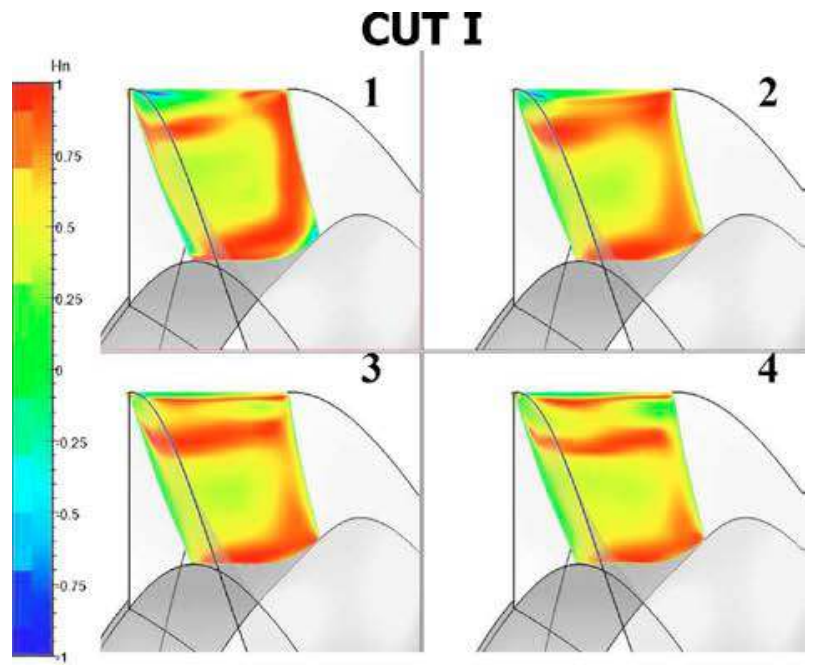

(a)

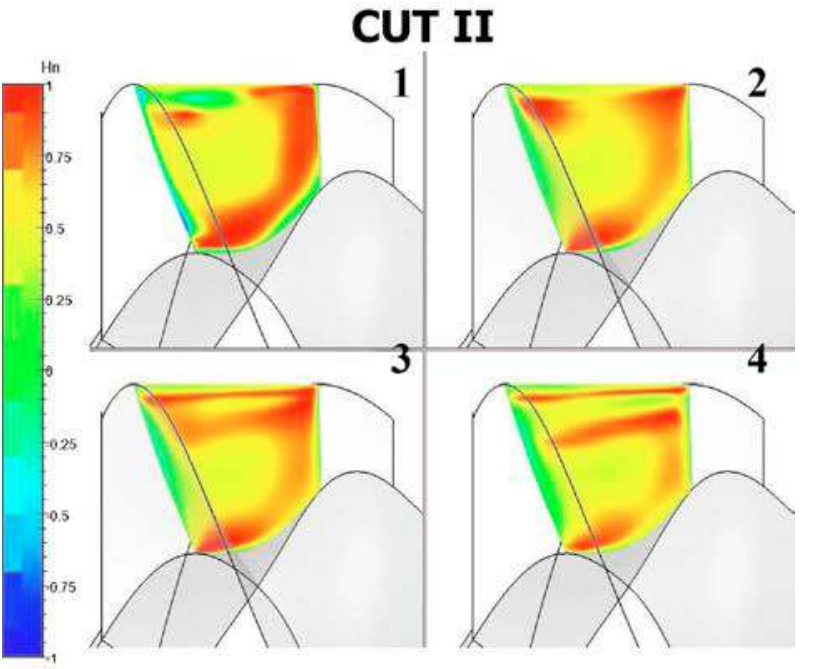

(b)

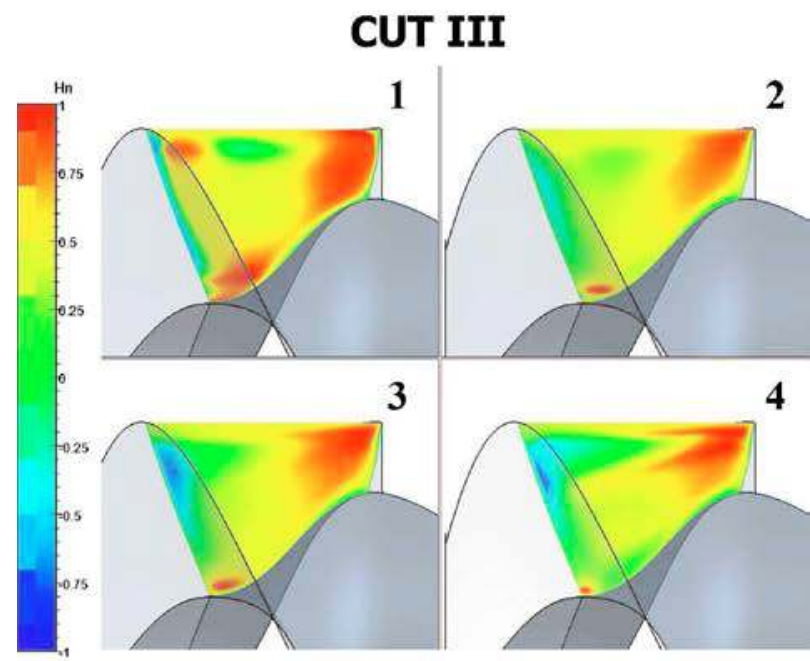

(c)

Fig. 16 Secondary flows: dimensionless relative helicity representation at three locations in the blade-to-blade channel: (a) Sec. 1, (b) Sec. 2, and (c) Sec. 3. Blue represents the maximum anticlockwise vorticity zone and red the maximum clockwise vorticity zone. The results are shown for the noncavitating condition (1) and typical cavitating regimes: $\tau^{*}=0.31, \tau^{*}=0.14$, and $\tau^{*}=0.11(2,3$, and 4$)$.

$$
H=\frac{W \cdot \operatorname{rot}(W)}{|W| \times|\operatorname{rot}(W)|}
$$

The normalized relative helicity indicates the value of the cosine of the angle between the velocity vector and the swirl vector. Consequently, the swirling centers will be associated with values +1 and -1 , the sign determining the direction of the rotation of the swirl. This quantity is drawn in Fig. 16 for the three defined sections during the breakdown, for the $1.4 Q n$ flow rate. The black color represents anticlockwise vorticity and yellow to white indicate clockwise vorticity.

In Sec. 1, the secondary flow in the rotating frame develops mainly along the blade suction side (white color), especially in the noncavitating condition. With the cavitation coefficient decrease, the clockwise vorticity on the suction side tends to be less important. A clockwise vorticity structure of low intensity is located on the pressure side. This behavior is due to the development of the boundary layer, which is less important on the pressure side than on the suction side because of the leading edge proximity on the pressure side. It is also observed that the flow is disturbed in the zone near the hub; the flow displays a high clockwise vorticity.

It is important to recall that tip leakage was not considered in the numerical simulations (which explains the absence of zones with a strong vorticity near the shroud).

In the noncavitating regime in Sec. 2 (Fig. 16(b)), we observe a clockwise vorticity (white color) on the suction side. For the cavitating conditions the secondary flows are similar to the structures observed in Sec. 1.

Finally, in Sec. 3 (Fig. 16(c)), in the noncavitating regime, the clockwise vorticity is located on the suction side and some regions of the anticlockwise vorticity appear close to the shroud on the pressure side. The vorticity effects are less important near the hub for this section due to the meridian curvature of this inducer.

In the analyzed sections in cavitating conditions, we observed some vorticity structures (white color) located near the shroud, which seem to be associated with the ringed vapor structure at the shroud (Figs. 11 and 12). Boundary layers are developed in the zones close to solid surfaces of the blades in the pressure side and suction side for the three different sections.

Figure 17 shows the distribution of the radial velocity in the three sections. For noncavitating conditions we observe a strong centrifugation of the boundary layers, mainly at the suction side (radial velocity is around $12 \mathrm{~m} / \mathrm{s}$ ), which is associated to the secondary structures observed from helicity analyses.

It is worth noting that comparisons between results obtained from grids V1, V2, and V3 indicate slight modifications on calculated secondary flows, as illustrated in Fig. 18.

4.5.2 Axial Velocity. In order to analyze the flow rate distribution in the inducer, results obtained for the $1.4 Q n$ flow rate concerning the axial velocity in the three sections previously defined (Fig. 14) are illustrated in Fig. 19. Results correspond to different $\tau^{*}$ values.

In Sec. 1 (Fig. 19(a)), a backflow zone near the shroud, characterized by negative axial velocities can mainly be observed. This zone is clearly observed in the meridian view by the analysis of the streamlines (Fig. 20). An anticlockwise vorticity (Fig. $16(a)$ ) can also be observed in this zone (Sec. 1, noncavitating condition).

The figures corresponding to Sec. 2 (Fig. 19(b)) show that the zones of the negative axial velocity are mainly near the hub. For the smallest values of the cavitation parameter, the appearance of zones of low velocities close to the shroud can be observed. For Sec. 3 (Fig. 19(c)), the negative axial velocity area is placed in the pressure side near the hub and it seems to be related to the high meridian curvature of the hub.

In a general overview, it can be noticed that the flow rate is not uniformly distributed in the channel and that axial velocities are more important at the blade suction side. 

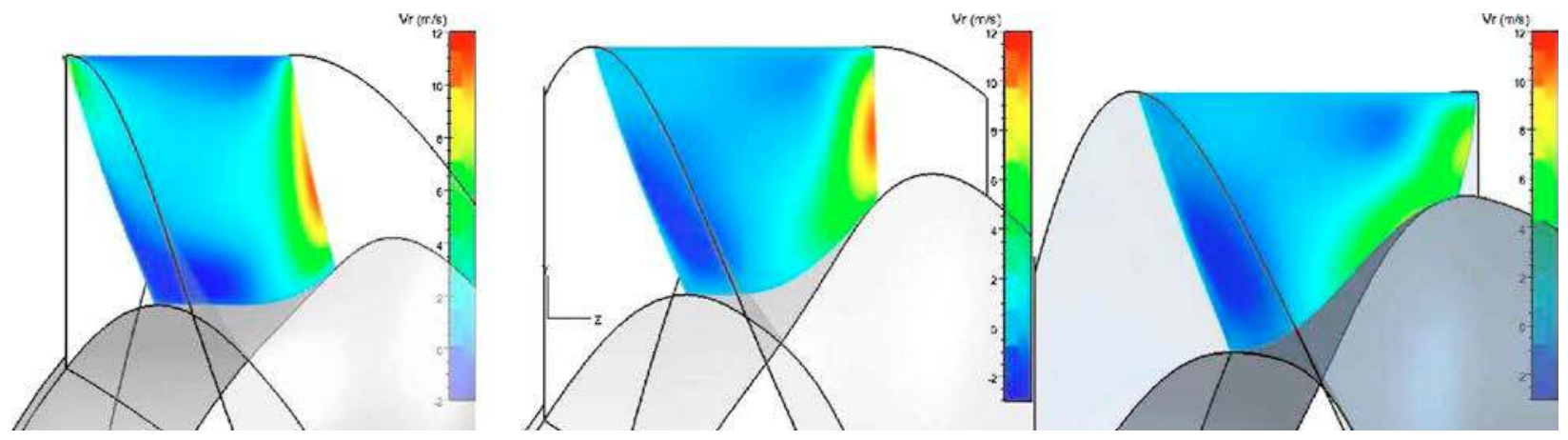

Fig. 17 Secondary flows: radial velocity evolution in the noncavitating regime in Secs. 1-3 for 1.4Qn

By analyzing results presented in Fig. 20, we observe that the backflow zone near the shroud tends to decrease during breakdown; the appearance of cavitation structures and the reduction of pressure gradients between the suction and pressure sides lead to a decrease in the thickness of the backflow area (in the meridian plane). The width (Fig. 19(a)) and the length (Fig. 20) of this zone are also modified by cavitation structures.

\section{Conclusion}

In the present work, 3D steady RANS computations were performed and compared with experiments concerning water cavitating flows in a three-blade inducer at different flow rates. In complement to prior works $[4,10,11]$, the main targets of the present study were to pursue model validation and to improve analyses of complex 3D cavitating flows in rocket engine turbopump inducers.

The qualitative development of the cavitation in the inducer was simulated and the extension of vapor near the shroud was favorably compared with experimental results. Experimental and numerical performance charts concerning three different flow rates were also compared. Thanks to recent numerical improvements, predictions of the inducer breakdown were adequate and much better than the ones presented in previous works [11].

Analyses of the numerical results provided interesting information about the power, blade load, and hydraulic efficiency evolution during head breakdown. Secondary flows were analyzed from dimensionless relative helicity maps corresponding to three different sections in the blade-to-blade channel. Moreover, local analyses of axial velocity fields enabled the determination of backflow zones and of the flow rate distribution in the inducer channels. For the considered cavitation number range, the study pointed out a relevant influence of the cavitation structures on the flow fields around the leading edge, near the shroud zone.

The methodology of analysis proposed in this paper showed that numerical simulations can provide useful information for the design of turbomachinery, and more particularly for inducers. Based on the experimental and numerical results obtained, the inducer geometry was improved. A new inducer was designed and is being tested for future works concerning mainly instable cavitation analyses and prediction.

Complementary works are also in progress, mainly

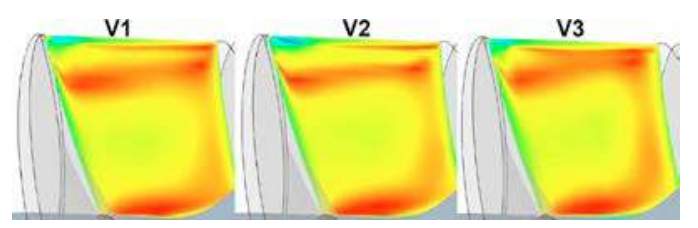

Fig. 18 Secondary flows: dimensionless relative helicity (Cut I) at $1.4 Q n$ at $\tau^{*}=0.24$. Representation of numerical results in Grids V1, V2, and V3.
- to improve the cavitation model to take into account thermodynamic effects, mainly in the case of cryogenic propellants $[25,26]$ and

- to implement and to apply a multigrid strategy to accelerate the convergence of cavitating flow calculations in inducer geometries. This work is very important for carrying out 3D unsteady calculations and for analyzing cavitation instabilities $[8,20,27]$.

It is worth underlining that further local and global experimental studies are also required in order to validate and calibrate the physical models applied in an improved way.

\section{Acknowledgment}

The authors wish to express their gratitude to French space agency CNES, to Snecma for their continuous support, and to Numeca International for their cooperation in the development of the numerical code. This research was also supported by a doctoral grant from the Los Andes University, Venezuela.

\section{Nomenclature}

$$
\begin{aligned}
& c_{\min }=\text { minimum speed of sound in the vapor/liquid } \\
& L_{\text {ref }}=\text { geometry reference length }(\mathrm{m}) \\
& P=\text { local static pressure }(\mathrm{Pa}) \\
& P_{\text {tot }}=P+\left(\rho V^{2} / 2\right) \text { local total pressure }(\mathrm{Pa}) \\
& P_{\text {vap }}=\text { vapor pressure }(\mathrm{Pa}) \\
& \text { power }=T \omega(\mathrm{W}) \\
& \text { power }^{*}=\text { power } / \text { power }_{\text {noncavitating dimensionless power }} \\
& Q=\text { flow rate }\left(\mathrm{m}^{3} / \mathrm{s}\right) \\
& Q n=\text { nominal flow rate }\left(\mathrm{m}^{3} / \mathrm{s}\right) \\
& T=\text { torque }(\mathrm{N} \mathrm{m}) \\
& V=\text { flow velocity }(\mathrm{m} / \mathrm{s}) \\
& V_{\text {ref }}=\text { reference velocity: tangential tip velocity }(\mathrm{m} / \mathrm{s}) \\
& W=\text { relative flow velocity }(\mathrm{m} / \mathrm{s}) \\
& \Delta P_{\text {tot }}=P_{\text {tot downstream }}-P_{\text {tot upstream }} \text { total pressure varia- } \\
& \Psi=\left(P_{\text {static }}^{\text {outlet }}-P_{\text {static }}^{\text {inlet }}\right) / \rho \cdot V_{\text {ref }}^{2} \text { shroud elevation } \\
& \rho=\text { fluid density }\left(\mathrm{kg} / \mathrm{m}^{3}\right) \\
& \rho_{l}, \rho_{v}=\text { liquid/vapor density }\left(\mathrm{kg} / \mathrm{m}^{3}\right) \\
& a=\left(\rho-\rho_{l}\right) /\left(\rho_{v}-\rho_{l}\right) \text { void ratio } \\
& \eta=\Delta P_{\text {tot }} Q / T \omega \text { hydraulic efficiency } \\
& \tau=P_{\text {tot }}^{\text {inlet }}-P_{\text {vap }} / \rho \cdot V_{\text {ref }}^{2} \text { cavitation parameter } \\
& \tau^{*}=(\tau / \text { const }) \text { dimensionless cavitation parameter } \\
& w=\text { angular velocity }(\mathrm{rad} / \mathrm{s}) \\
& y^{+}=\text {dimensionless distance to the wall }
\end{aligned}
$$




\section{CUT I}
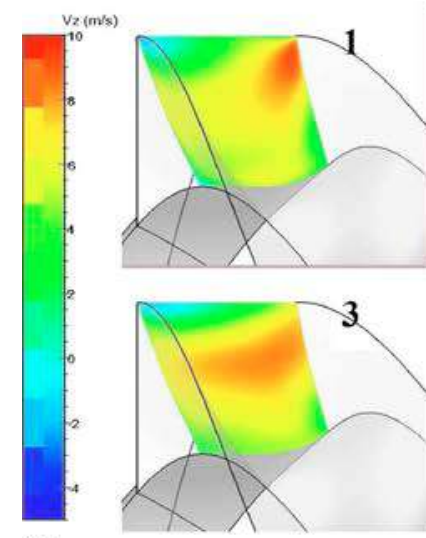

(a)

\section{CUT II}
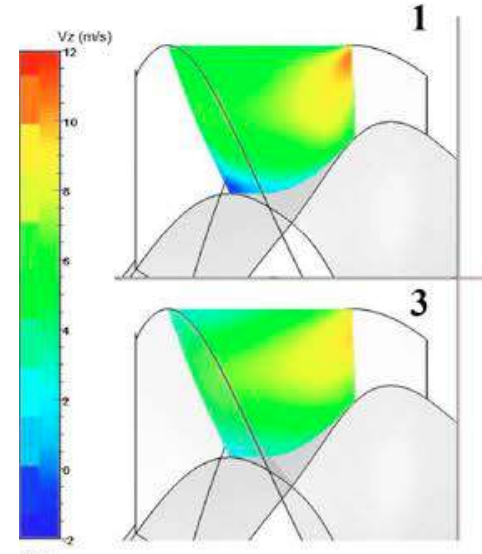

$1+1-2$

(b)

\section{CUT III}
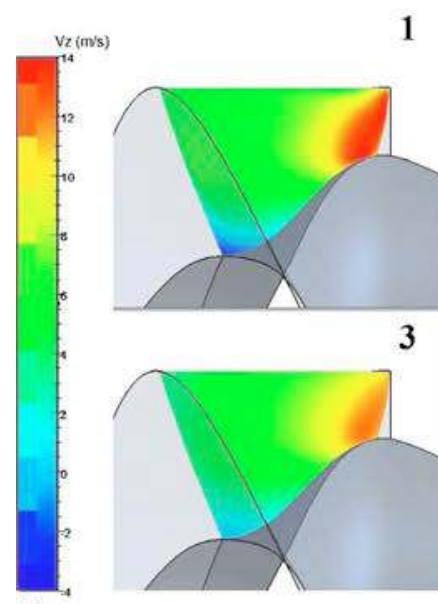

1
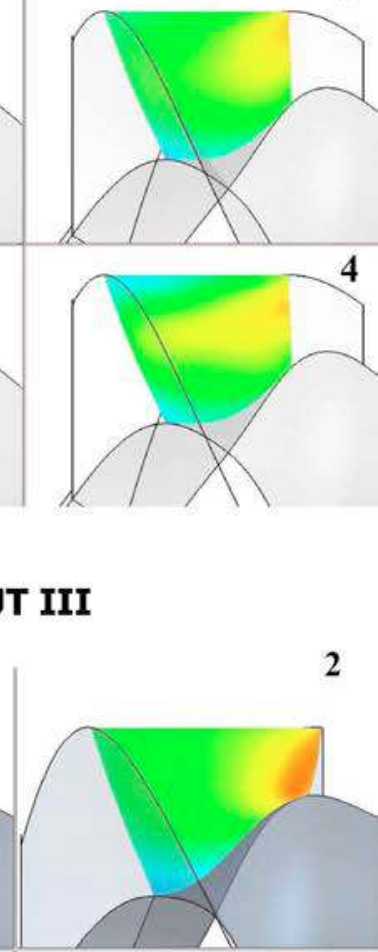

4

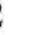


"Numerical Model to Predict Unsteady Cavitating Flow Behaviour in Inducer Blade Cascades," ASME J. Fluids Eng., 129(2), pp. 128-135.

[21] Jameson, A., Schmidt, W., and Turkel, E., 1981, "Numerical Solution of the Euler Equations by Finite-Volume Methods Using Runge-Kutta Time Stepping Schemes," AIAA Paper No. 81-1259.

[22] Yang, Z., and Shih, T. H., 1993, "A k-e Model for Turbulence and Transitional Boundary Layer," Near-Wall Turbulent Flows, R. M. C. So, C. G. Speziale, and B. E. Launder, eds., Elsevier, New York, pp. 165-175.

[23] Coutier-Delgosha O., Fortes-Patella R., Reboud J. L., Hakimi N., and Hirsch C., 2005, "Stability of Preconditioned Navier-Stokes Equations Associated With a Cavitation Model," Comput. Fluids, 34(3), pp. 319-349.

[24] Pouffary B., Fortes-Patella R., Reboud J. L., and Lambert P. A., 2008, "Nu- merical Analysis of Cavitation Instabilities in Inducer Blade Cascade," ASME J. Fluids Eng., 130(4), to be published.

[25] Rolland, J., Boitel, G., Barre, S., Goncalves, E., and Fortes Patella, R., 2006 "Inducer Experiments and Modelling of Cavitating Flows in Venturi: Part I: Stable Cavitation," Proceedings of the Symposium CAV2006, Wageningen, The Netherlands, Sep. 11-15.

[26] Rolland, J., 2008, "Modélisation Des Écoulements Cavitants Dans les Inducteurs de Turbopompes: Prise en Compte des Effets Thermodynamiques," Ph.D thesis, Institut National Polytechnique de Grenoble, France.

[27] Horiguchi, H., Watanabe, S., Tsujimoto, Y., and Aoki, M., 2000, "A Theoretical Analysis of Alternate Blade Cavitation in Inducers," ASME J. Fluids Eng., 122(2), pp. 156-163. 